\title{
Prospectiva archivística: nuevas cuestiones, enfoques y métodos de investigación científica
}

\author{
M. Paz Martín-Pozuelo*
}

Resumen: La archivística, fuertemente influenciada por los cambios sociales y tecnológicos, experimenta en la actualidad transformaciones tan profundas que su propia naturaleza se está viendo modificada. Desde una breve introducción a los estudios de futuro en este artículo se define la prospectiva archivística como la investigación que se plantea y se desarrolla para dar respuesta a los interrogantes y la incertidumbre respecto al futuro de los archivos y de la archivística así como al estudio de los factores de cambio que determinarán ese futuro. Se reflexiona y se discute acerca de sus fundamentos, las cuestiones que debe resolver, sus diferentes campos de aplicación y los métodos más apropiados. El artículo finaliza con una propuesta acerca de los resultados de una adecuada aplicación de la prospectiva archivística: documentos de consenso e indicadores.

Palabras clave: Archivística, prospectiva, prospectiva archivística, investigación archivística, análisis prospectivo, métodos de investigación, técnicas de consenso, vigilancia, vigilancia tecnológica.

\section{Prospective archival science: new issues and methods for archival research}

Abstract: Archival science, which is highly influenced by social and technological change, is presently in the midst of a profound transformation that affects its very nature. After a brief introduction to future studies, prospective archival science is defined as the research posed and conducted to respond to queries and uncertainties respecting the future of archives and archival science, as well as the study of factors of change that will determine that future. This is followed by a reflection on the fundamentals of this discipline, the questions to be addressed, the fields of application, and the most suitable methods. The article concludes with a proposal for the appropriate application of prospective archival science: consensus documents and indicators.

Keywords: Archival science, prospective archival science, archival research, prospective analysis, consensus techniques, monitoring, technological monitoring.

\footnotetext{
* Departamento de Biblioteconomía y Documentación. Universidad Carlos III de Madrid. Correo-e: ppozuelo@bib.uc3m.es.

Recibido: 1-6-09; 2. ${ }^{\text {a }}$ versión: 1-10-09; aceptado: 25-1-10.
} 


\section{Planteamiento}

Como disciplina que estudia la documentación que producen los organismos y las sociedades, la archivística, fuertemente influenciada por los cambios sociales y tecnológicos, experimenta en la actualidad transformaciones tan profundas que su propia naturaleza se está viendo modificada. Algunas investigaciones revelan la necesidad de estudios que evidencien el impacto real de estos cambios (Gilliland-Swetland, 2000; Duranti, 2000; Cook, 2001; Martín-Pozuelo, 2002; Borglund, 2005). Para ello, esto es, para estudiar el impacto de estas transformaciones, las nuevas cuestiones así como la incertidumbre que el futuro más y menos inmediato está provocando, la archivística necesita desarrollar nuevos enfoques y herramientas de investigación. La finalidad de este artículo es, precisamente, la de presentar la prospectiva archivística como nuevo enfoque de investigación científica en y para el estudio del futuro de los archivos, de sus profesionales y de la ciencia que los estudia. Se propone también discutir acerca de los métodos más adecuados para su aplicación. Desde una aproximación a los estudios de futuro se definen aquí las cuestiones que éstos plantean para la archivística.

En su conjunto las ideas y las propuestas recogidas en este artículo parten de un presupuesto básico y fundamental: la necesidad de actuar cuanto antes sobre el futuro de la archivística, el convencimiento de que la prospectiva es la propuesta científica más adecuada para conocer los factores y puntos clave de ese futuro, así como la herramienta metodológica que facilita y hace posible la reflexión colectiva sobre el archivo, la archivística y sus profesionales.

\section{Introducción. La prospectiva y los estudios de futuro}

No existe por el momento una definición que podamos referir como de absoluto consenso respecto a la prospectiva y los estudios de futuro. En lo que sí parece existir unanimidad es en la consideración acerca de lo que no son estos estudios ni es la prospectiva. Ni la prospectiva ni los estudios de futuro son técnicas adivinatorias. En ningún caso nos permiten adivinar el futuro ni en su conjunto ni en sus partes. Estos estudios son, en realidad, un grupo de disciplinas cuya metodología de análisis basada en la observación sistemática, el consenso colectivo y la anticipación, les dota de un carácter muy cercano a lo científico.

No es éste el lugar para presentar el origen ni el desarrollo de estas disciplinas (Medina Vásquez y Ortegón, 2006) que, por otra parte, tienen en la actualidad un notable desarrollo. Como contexto de esta exposición y sin ánimo de exhaustividad haré un brevísimo recorrido con el fin de dejar sentada la finalidad de estos estudios como marco teórico que justifica la disciplina que aquí se propone: la prospectiva archivística.

En términos muy globales y siguiendo a Medina Vázquez y Ortegón, los estudios de futuro pueden situarse al inicio del siglo xx cuando en 1907 Gilfillan, especialista en ciencias sociales sugiere que se denomine Melontología al estudio 
de las civilizaciones futuras (Medina Vásquez y Ortegón, 2006). Sin embargo, son varios los autores que lo hacen coincidir con 1957 cuando Gastón Berger crea la voz prospectiva por oposición al término retrospectiva y la define como "la ciencia que estudia el futuro para poder influir en él" (Medina Vásquez y Ortegón, 2006; Waardenburg, 1999) Desde entonces hasta hoy todos los intentos definitorios se reducen a insistir sobre la misma idea: conocer el futuro para poder influirlo.

TABLA I

Evolución del término sobre estudios de futuro

\begin{tabular}{|c|c|c|}
\hline Año & Autor & Descripción \\
\hline 1907 & Gilfillan & $\begin{array}{l}\text { Sugiere que se llame Melontología el estudio de las } \\
\text { futuras civilizaciones. }\end{array}$ \\
\hline 1943 & O. K. Flechtheim & $\begin{array}{l}\text { Propone el término Futurology para la búsqueda una } \\
\text { lógica al futuro. }\end{array}$ \\
\hline 1957 & Gaston Berger & $\begin{array}{l}\text { Crea la voz Prospectiva por oposición al término Re- } \\
\text { trospectiva. }\end{array}$ \\
\hline 1966 & & Se funda la World Future Society. \\
\hline 1967 & Bertrand de Jouvenel & $\begin{array}{l}\text { Propone Futuribles para el estudio de los futuros posi- } \\
\text { bles. }\end{array}$ \\
\hline 1970 & Erich Janstch & Utiliza tecnological forestcasting. \\
\hline 1971 & Fred Polak & Propone prognostic. \\
\hline 1973 & UNESCO & Nace la World Futures Studies Federation. \\
\hline 1974 & $\begin{array}{l}\text { El secretariado sueco para } \\
\text { los estudios de futuro }\end{array}$ & Propone Futures Studies en lugar Futures research. \\
\hline $\begin{array}{c}1975 \\
\text { en } \\
\text { adelante }\end{array}$ & & $\begin{array}{l}\text { Future analysis, futurics, futures field, probabilistics, } \\
\text { forward studes, future planning, futurography, } \\
\text { antrospectrunity, projective research, short and long } \\
\text { range planning, projections, predictive studies, futury } \\
\text { (como correspondiente a bistory), futory (comofuture } \\
\text { más history), alternative analysis, options analysis, } \\
\text { decision option analysis, alternative in futures, } \\
\text { prospectivism, fustory (por bistoria futura), futurist o } \\
\text { futuristic studies. }\end{array}$ \\
\hline 1976 & Daniel Bell & Utiliza por primera vez la voz prognosis. \\
\hline $\begin{array}{l}\text { Mediados } \\
\text { de los } 80\end{array}$ & & Foresight, para la identificación de nuevas tecnologías. \\
\hline
\end{tabular}

Fuente: Elaboración propia desde Medina Vásquez, J. y Ortegón, E. (2006).

En el mismo sentido y desde la literatura de anticipación el concepto de futuro presenta una evolución diferente en las dos demarcaciones territoriales don- 
de finalmente se desarrolla: Europa y Estados Unidos. En el cuadro que sigue se recogen las principales tendencias de estos estudios en ambos espacios geográficos.

TABLA II

Literatura de anticipación siglos XIX $y$ XX

\begin{tabular}{l|l}
\hline \multicolumn{1}{c|}{ Estados Unidos } & \multicolumn{1}{c}{ Europa } \\
\hline Planificación de largo plazo (50) & Futurología (40) \\
Investigación de futuros (60) & Prospectiva (50) \\
• Pronóstico tecnológico (60) & • Prospectiva estrategica (70) \\
- Planificación por escenarios (70) & • Previsión humana y social (70) \\
\hline
\end{tabular}

Fuente: Elaboración propia desde Medina Vásquez, J. y Ortegón, E. (2006).

De un modo excepcionalmente resumido podemos afirmar que los estudios de futuro persiguen establecer la diferencia y llegar así a la comprensión de los tres tipos de futuro: posible, probable y deseable (Bell, 1997).

La discusión acerca del carácter científico de la prospectiva está presente en diferentes estudios (Godet, 2004). Con Jordi Serra coincidimos en señalar que la prospectiva es en realidad, una ciencia sin objeto que se mueve entre la necesidad de predecir lo que puede ocurrir y el deseo de inventar el mejor futuro posible (Serra,1997) Hay, sin embargo, una serie de rasgos que nos permiten identificar con cierta claridad el tipo de reflexión que exige. Siguiendo a Eleanora Masini (2000), en una presentación global se concretarían en los que, a continuación, se relacionan.

a) Transdisciplinaridad: por cuanto en el futuro inciden aspectos muy variados que obligan a un análisis desde muy diferentes áreas de conocimiento. En consecuencia, ningún estudio sobre el futuro puede progresar sin la colaboración y la conexión de otras disciplinas siempre conectadas.

b) Complejidad: en tanto ha de considerar el conjunto de aspectos conectados que influyen o pueden influir sobre el futuro.

c) Globalidad: en toda reflexión prospectiva hay un objetivo claro: establecer las relaciones entre lo interno y lo externo y los diferentes momentos: pasado, presente y futuro.

d) Normatividad: implica un querer ser de la sociedad en tanto se propone futuros deseables.

e) Cientificidad: radica en el método en tanto su objeto esto es, el futuro, no es objeto experimental. También en el rigor con que se formulen las hipótesis y en la validación de sus resultados.

f) Dinamicidad: incluye e implica una renovación constante frente a los cambios.

g) Participación: está fundamentada en la participación y el debate. 
En su obra La caja de herramientas Michel Godet insiste sobre la necesidad de distinguir entre tres conceptos claves: planificación, prospectiva y estrategia. Asegura además que se trata de tres conceptos en muy estrecha interrelación (Godet, 2000). Dos años más tarde Gavigan sitúa la prospectiva en el campo de intersección de tres diferentes disciplinas: la planificación estratégica, los estudios de futuro y el análisis de políticas (Gavigan, 2001).

Más arriba he señalado que la prospectiva no es futurología y, en consecuencia, no ayuda a predecir el futuro, pero lo que no he dicho es que sí nos ofrece la posibilidad de construirlo. Así, en la medida en que ese futuro se prevea más o menos incierto o probable la prospectiva será en mayor o menor medida recomendable y su utilidad crecerá o decrecerá en idéntica proporción a las incertidumbres que el futuro provoque. En este sentido y partiendo del hecho indiscutible de que el futuro es siempre incierto, la prospectiva se fija en que el grado de incertidumbre respecto a ese futuro lo marcan la serie de factores que puedan influirle. A mayor número de factores mayor incertidumbre. La aceleración del cambio y, en consecuencia, el incremento de la incertidumbre constituyen la verdadera razón por la que la prospectiva resulta necesaria.

Por lo que hace a sus campos de aplicación desde la literatura especializada se identifican tres principales tipos de prospectiva: tecnológica, territorial, y organizacional. Existen, así mismo, tantos tipos de prospectiva como áreas o especialidades sobre las que ésta puede tener una determinada aplicación. Un análisis de la bibliografía especializada nos ha permitido construir la tabla III que recoge los principales estudios por autor o autores, en relación con las principales áreas sobre las que se aplica la prospectiva.

Desde un análisis necesariamente somero parece lógico concluir que la prospectiva va avanzando en la ocupación de áreas cada vez más diversas. Lógico es pensar también que tenga presencia en aquéllas en las que el futuro provoca mayor número de incertidumbres. Es el caso de la archivística. Las líneas que siguen están dedicadas a la exposición de la prospectiva archivística, esto es la aplicación a la archivística de las técnicas que nos hacen posible la anticipación sobre el futuro de los archivos y de la disciplina que los estudia.

\section{La prospectiva archivística: definición y fundamentos}

\subsection{Definición y objetivos de la prospectiva archivística}

Por prospectiva archivística entiendo la investigación que se plantea y se desarrolla para dar respuesta a los interrogantes y la incertidumbre respecto al futuro de los archivos y de la archivística, así como al estudio de los factores de cambio que determinarán ese futuro. Dicho de otra manera, la prospectiva archivística no es otra cosa que la aplicación al estudio de los archivos de las técnicas que nos hacen posible el conocimiento y la anticipación sobre su futuro con el fin de diseñar estrategias para promover su calidad y desarrollo integral. 
TABLA III

Áreas de aplicación actual de la prospectiva y principales autores que la estudian

\begin{tabular}{l|l}
\hline \multicolumn{1}{c|}{ Área de conocimiento } & \multicolumn{1}{c}{ Autor/es } \\
\hline \multirow{2}{*}{ ADMINISTRACIÓN } & $\begin{array}{l}\text { Ackoff, Russell, L. (1979). } \\
\text { Bailly, Jean-Paul (1998). } \\
\text { Drucker, Peter F. (2003). } \\
\text { Jacquard, Albert; Kahn, Axel (2001). }\end{array}$ \\
\hline DEMOGRAFÍA & La revue de la Prospective Social (2002). \\
\hline \multirow{2}{*}{ ECOLOGÍA } & $\begin{array}{l}\text { Bourg, Dominique; Scheler, Jean-Louis (2001). } \\
\text { García, Ernest (2004). } \\
\text { Jancovici, Jean-Marc (2002). } \\
\text { Gallissot, Joël (2004). } \\
\text { Michalski, Wolfgang; Miller, Riel; Stevens, Barrie y otros (1999). } \\
\text { Leff, Enrique (2004). }\end{array}$ \\
\hline ECONOMÍA & $\begin{array}{l}\text { Clarke, Rory; Durand, Martine; Pilat, Dirk et Torres, Raymond (2001). } \\
\text { Heilbroner, Robert (1996). } \\
\text { Michalski, Wolfgang; Miller, Riel; Stevens, Barrie, y otros (1999). }\end{array}$ \\
\hline HISTORIA & Cacace, Incola (2002). \\
\hline Healy, Tom; Coté, Sylvain, y otros (2001).
\end{tabular}

Fuente: Elaboración propia.

En su relación con la archivística, la prospectiva es una metodología cuyos rasgos podemos concretar como sigue:

a) Se fundamenta en la observación sistemática, la vigilancia y la inteligencia competitiva.

b) Se construye desde el debate y el consenso.

c) Facilita y sistematiza la reflexión colectiva.

d) Para su aplicación y análisis requiere de la colaboración de diferentes áreas de conocimiento lo que la dota de un carácter multidisciplinar.

e) Facilita la anticipación.

f) Está referida a largo plazo.

Los objetivos de la prospectiva archivística pueden concretarse del modo que sigue:

- Anticipar los cambios o rupturas de la archivística con respecto al futuro.

- Identificar las nuevas tendencias. 
- Conocer las actitudes y el poder de la comunidad archivística para hacer frente a esos cambios y esas nuevas tendencias.

- Conocer la capacidad de la comunidad archivística para dar respuestas a los posibles problemas de futuro.

- Potenciar la comunicación con todos los sectores y colectivos implicados en el proceso archivístico.

- Promover la comunicación entre todos los miembros de la comunidad archivística.

- Definir puntos débiles y fuertes de la disciplina y su entramado tanto teórico como práctico.

- Plantear retos inmediatos y futuros en el desarrollo de la archivística.

- Plantear el estudio de escenarios de futuro para el desarrollo social de la archivística.

\subsection{Fundamentos}

Las ideas y conceptos sobre los que, entiendo, se debe fundamentar la investigación prospectiva archivística están siendo objeto de mi atención en una investigación dedicada al estudio de las relaciones de los archivos con la sociedad. Se recogen aquí aquellas ideas que tienen una clara relación con la investigación prospectiva.

\section{A. La anticipación o la fuerza de las hipótesis}

Considerada como "proyección" de un conocimiento futuro, la anticipación se entiende aquí como un conjunto de ideas claves o ideas fuerza, esto es, de hipótesis que constituyen la base a partir de las cuales se plantea la investigación prospectiva.

Toda investigación prospectiva en archivística debe considerar una o varias de las siguientes hipótesis o ideas fuerza:

- Idea fuerza 1. La existencia de archivos es uno de los principios básicos de democracia, transparencia y desarrollo humano.

- Idea fuerza 2. Los avances tecnológicos permiten un uso mayor, más generalizado y mejor de los archivos.

- Idea fuerza 3. La comunicación de los archivos con la sociedad exige el desarrollo de estrategias basadas en reflexiones colectivas que implican a todos los colectivos sin excepción.

- Idea fuerza 4. Las instituciones, organizaciones y la humanidad en su conjunto necesitan un aumento de la accesibilidad a los archivos. Solo un despliegue necesario de medios en estas unidades de información hará posible la respuesta satisfactoria a esta necesidad. 
- Idea fuerza 5. Urge crear un marco de desarrollo archivístico que incluya e interrelacione las cuestiones relativas a la producción y la gestión de documentos, así como a la socialización de sus contenidos.

- Idea fuerza 6. Urge así mismo el desarrollo de políticas públicas de apoyo decidido a los archivos y sus profesionales.

- Idea fuerza 7. El futuro trae incertidumbres en tanto las tecnologías se imponen mientras los cambios sociales siguen creciendo. Solo un conocimiento profundo de este futuro nos permitirá actuar sobre él.

- Idea fuerza 8. Para encarar el futuro de los archivos son necesarios ejercicios de observación y vigilancia sistemática y rigurosa.

- Idea fuerza 9. Son necesarios también programas de I+D e I+T para abordar el desarrollo tecnológico en los archivos.

- Idea fuerza 10. La colaboración académica- profesional, esto es, el acercamiento entre las dos fuerzas a partir de las que se construye el pensamiento archivístico, la formación y la investigación por un lado y la práctica profesional por el otro.

En su conjunto las hipótesis (ideas fuerza) definidas revelan la necesidad de un diálogo sistemático y organizado.

\section{B. El diálogo en la construcción social del archivo}

Más arriba he señalado que la archivística se entiende aquí como la ciencia que estudia la documentación que generan organizaciones y sociedades. Su construcción futura pasa entonces por el estudio de la comprensión de las relaciones de la archivística, los archivos y sus profesionales con la sociedad. Toda su investigación debe partir necesariamente del diálogo social, esto es el diálogo con sus diferentes colectivos, de un modo especial con aquéllos para los que el archivo representa un elemento activo. También con el de aquellos actores directos de la praxis archivística: responsables de políticas archivísticas, productores de documentos, archiveros y usuarios. Todos ellos se han agrupado en cuatro diferentes grupos, a saber:

a) Grupos de decisión, en los que se incluyen todos aquellos miembros de la sociedad capacitados para adoptar decisiones que luego han de ser llevadas a término, políticos principalmente. En el estudio del futuro archivístico tienen un interés especial todos aquellos individuos cuyas decisiones impactan directamente sobre los archivos. No considero aquí solamente los políticos con capacidad y competencias en materia archivística, incluyo también todos aquellos individuos cuyas decisiones finalmente implican a los archivos: alcaldes, secretarios de ayuntamientos, concejales, etc.

El diálogo con este grupo tiene el objetivo principal de constatar el grado de conocimiento sobre el archivo y sus profesionales, su funcio- 
namiento, organización, con aquellos de los que depende su infraestructura y dotación principalmente. También su planificación a corto, medio y largo plazo.

b) Grupos de ejecución. Se entienden incluidos en este grupo todos aquellos individuos cuya principal función es hacer cumplir las decisiones de otros. En el ámbito archivístico estamos hablando fundamentalmente de los archiveros encargados de aplicar las directrices y las direcciones adoptadas por los diferentes miembros de los grupos de decisión.

El diálogo con este grupo tiene el objetivo principal de conocer la realidad de los archivos desde el testimonio directo de sus protagonistas, esto es, de aquellos individuos que tienen la información más cualificada sobre los problemas, los inconvenientes, los retos, las oportunidades y las amenazas de los archivos y sus relaciones con la sociedad. También para constatar la distancia entre la teoría y la práctica.

c) Grupos de opinión, se incluyen aquí todos los miembros de la sociedad que por su actividad y/o estima social tienen la capacidad de generar opinión: intelectuales y comunicadores principalmente.

Con ellos el diálogo tiene una doble finalidad. Por un lado conocer desde sus testimonios la percepción que tienen del archivo como fuente de información y como herramienta de poder. Por el otro, obtener información directa sobre su percepción de la sociedad con el fin de concretar las razones sobre el interés o desinterés que la misma tiene acerca del archivo.

d) En un último grupo quedan incluidos todos los miembros de la sociedad que consideramos usuarios de archivo, bien que este uso sea más o menos habitual o bien que lo sea esporádico. En ambos casos se consideran motivos de gestión, toma de decisiones, investigación, referencia, o de mera curiosidad.

En ocasiones y debidamente organizados se ha de contemplar la posibilidad de que éstos sean abiertos al público con el fin de multiplicar las miradas cualitativas. También para generar, al tiempo que investigamos, una curiosidad por el archivo, el archivero y la archivística.

\section{Los juicios colectivos y el consenso científico}

El debate acerca del valor científico de las denominadas técnicas de consenso sigue siendo un debate abierto (Pérez Andrés, 2000). Es cierto que el carácter colectivo no confiere a los juicios un valor científico, sin embargo, tienen el valor de representar de algún modo una preocupación colectiva. En el marco de la investigación científica tienen el valor de contribuir a la definición de problemas nuevos sobre los que nunca antes se tenía absolutamente ninguna noticia. En consecuencia, resultan fundamentales en el ámbito de la investigación prospectiva en tanto, como ya he dicho más arriba, su objeto no es otro que el conocimiento y la anticipación sobre su futuro. 
Las cuestiones básicas sobre las que la prospectiva archivística ha de buscar el consenso se concretan en las siguientes:

- ¿Qué aspectos afectan más a los archivos?

- ¿Cuáles lo hacen ahora?

- ¿Cuáles lo hacen ahora de un modo especial?

- ¿Cuáles afectarán en un futuro?

- ¿Cuáles lo harán de una manera más importante?

- ¿Cuáles de los que afectan ahora dejarán de afectar en el futuro?

Cada una de estas preguntas ha de ser formulada de un modo conveniente y diferente en los distintos campos de la archivística. Pasamos a desarrollarlo en los epígrafes que siguen.

\section{Tipología y campos de aplicación de la prospectiva archivística}

Las incertidumbres sobre el futuro de la archivística son muy diferentes. En consecuencia, la prospectiva archivística puede aplicarse a diferentes campos por lo que proponemos diferentes tipos.

TABLA IV

Tipología y campos de aplicación de la prospectiva archivística

\begin{tabular}{l|l}
\hline \multicolumn{1}{c|}{ Denominación } & \multicolumn{1}{c}{ Finalidad } \\
\hline PROSPECTIVA CIENTÍFICA & Futuro científico de la archivística. \\
\hline PROSPECTIVA TECNOLÓGICA & Futuro tecnológico de la archivística. \\
\hline PROSPECTIVA SOCIAL & Futuro de la relación archivos y sociedad. \\
\hline PROSPECTIVA PROFESIONAL & Futuro del profesional de la archivística. \\
\hline
\end{tabular}

Pasamos ahora a presentar cada uno de ellos con detalle de cuestiones que suscitan y objetivos a partir de los que se construye su enfoque particular.

\subsection{Prospectiva científica: Estudio del futuro científico de la archivística}

La prospectiva científica tiene su objeto principal en el estudio del desarrollo científico de la archivística: el futuro de la ciencia, sus relaciones con otras ciencias, grupos, líneas y proyectos de investigación, inversión y apoyo a la investigación. 


\section{Cuestiones}

- ¿Está la teoría archivística preparada para encarar el futuro?

- ¿Le servirán los conceptos y principios en los que hoy fundamenta su praxis?

- ¿Debe ampliar su campo de relaciones científicas?

- ¿Sus actuales relaciones serán suficientes en un futuro?

- ¿Con qué otras áreas de conocimiento su relación es necesaria, recomendable y/o imprescindible?

- ¿Cuáles han de ser sus nuevos campos y líneas de investigación?

- ¿Qué capacidades científicas ha de tener el futuro profesional?

- ¿Cómo se formará el futuro investigador en archivística?

- ¿Qué proyectos de investigación debe emprender para encarar el futuro?

- ¿Cómo, dónde se difundirán los resultados?

- Las publicaciones actuales ¿son suficientes?, ¿tienen la calidad necesaria?, ¿la suficiente difusión?

- ¿De qué recursos disfrutará? ¿Cómo, dónde, de quién ha de buscarlos?

- ¿Con qué apoyo político debe contar su I+D?

\section{Objetivos}

- Revisar los actuales conceptos y principios en los que fundamenta hoy su campo teórico.

- Revisar los procesos, procedimientos en que fundamenta su metodología.

- Evaluar la investigación en el área de la archivística: ¿adecuados objetivos?

- Detectar nuevos campos de investigación.

- Proponer políticas de apoyo a su desarrollo científico.

\subsection{Prospectiva social: el futuro de las relaciones de la archivística con la sociedad}

Para el estudio del desarrollo social del archivo, del futuro de la convivencia y la inserción de los archivos en la sociedad, de la percepción pública, impacto social, tratamiento informativo, impacto mediático de la archivística.

\section{Cuestiones}

- ¿De qué manera afectarán los cambios sociales a la organización del archivo?

- ¿Tendrán la misma estructura administrativa?

- ¿Tendrán la misma organización?

- ¿Seguirá existiendo la misma necesidad del archivo?

- ¿Regirán las mismas normas sociales?

- ¿Seguirán las mismas normas de acceso?

- ¿Idénticas normas de transparencia?

- ¿Surgirán nuevos tipos de usuarios?

- ¿Surgirán nuevos modelos de comunicación? 


\section{Objetivos}

La prospectiva social aplicada a la archivística tiene una clara finalidad: la de construir a partir de un diálogo plural una imagen integral y a largo plazo, de los archivos y su convivencia con la sociedad. Para ello la prospectiva debe permitirnos alcanzar los siguientes objetivos:

- Mayor visibilidad y comprensión de los retos futuros de los archivos.

- Mayor apropiación de los archivos como bien público.

- Apropiación de los archivos como elemento clave del desarrollo social integral.

- Desarrollo de la capacidad de anticipación de fenómenos cambiantes de incidencia en el ámbito de los archivos.

- Fortalecimiento de herramientas de seguimiento de la evolución de los archivos.

- Fomento de relaciones:

a) docentes de archivística-archiveros,

b) archiveros-archiveros,

c) archiveros-profesionales de otras áreas afines,

d) archiveros y docentes-políticos y otros representantes de la sociedad.

- Aportes a la construcción de una visión compartida de archivos, memoria y derecho a la información.

- Influenciar las políticas públicas de información en las políticas públicas archivísticas.

- Analizar las implicaciones de la información archivística en sectores como justicia, salud, educación, cultura, ciencia y tecnología, seguridad social, urbanismo, etc.

- Obtener indicadores que nos permitan la medición y las representaciones sociales y la comunicación en materia de archivos.

\subsection{Prospectiva tecnológica: estudio del futuro tecnológico de la archivística}

Para el estudio del desarrollo tecnológico del archivo: futuros entornos de creación de documentos, futuros soportes documentales, futuras soluciones a la conservación, futuras herramientas tecnológicas de comunicación.

\section{Cuestiones}

- ¿Cuáles de las tecnologías emergentes presentes hoy en los archivos serán una realidad el día de mañana?

- ¿Qué avances revolucionarios incorporarán los futuros sistemas?

- ¿Cómo decidir qué líneas de desarrollo requieren un apoyo institucional? 


\section{Objetivos}

- Explorar los posibles futuros de los avances en tecnología en relación con la gestión de documentos y archivos.

- Identificar factores emergentes que originen y produzcan avances en las relaciones entre tecnologías y archivos.

- Crear una herramienta clave a la hora de definir políticas y planes de I+T en archivos.

- Definir futuros espacios de desarrollo profesional.

- Definir capacidades futuras de los profesionales.

\subsection{Prospectiva profesional: el futuro profesional de archivos}

Para el estudio del desarrollo profesional, esto es, el futuro del profesional de la archivística y de los archivos, futuros entornos de desarrollo profesional y futuros entornos de formación.

\section{Cuestiones}

- ¿Está el profesional de archivos preparado para afrontar el futuro?

- ¿Cuáles son sus actuales debilidades?

- ¿Cuáles sus fortalezas?

- ¿Nueva formación?

- ¿Cómo cualificarla?

- ¿Qué habilidades debe reforzar?

- ¿Qué nuevas habilidades debe adquirir?

\section{Objetivos}

- Factores clave que afectan a su desarrollo.

- Identificación de tendencias de cambio.

- Diseño y creación de escenarios.

- Retos inmediatos.

- Retos futuros.

\subsection{Otros campos de aplicación}

Se pueden plantear tantos análisis prospectivos parciales como aspectos incidan o puedan incidir sobre el futuro de la disciplina. Entre otros, los temas que atañen al futuro de los archivos podemos contemplar:

- Dinámicas demográficas.

- Dinámicas sociales.

- Dinámicas administrativas. 
- Dinámicas políticas.

- Dinámicas judiciales.

- Dinámicas sanitarias.

- Futuro de los profesionales.

- Futuro de la enseñanza.

- Futuro de la investigación.

- Futuro de la formación.

- Potencial de los archivos.

- Innovación en los archivos.

- Política archivística.

- Redes y colaboración entre archiveros.

\section{El proceso de investigación en la prospectiva archivística. Métodos y herramientas}

\subsection{La observación y la vigilancia como punto de partida}

Siguiendo a Godet (2000) la prospectiva se plantea para responder a cuatro interrogantes respecto del futuro:

- ¿Qué puede ocurrir?

- ¿Qué puedo hacer yo?

- ¿Qué voy a hacer?

- ¿Cómo voy a hacerlo?

De ninguna manera sería posible responder a las preguntas arriba expuestas sin tener un conocimiento profundo sobre los hechos del entorno que afectan o pueden afectar al objeto de análisis sobre el que planteamos la prospectiva. Este conocimiento sólo es posible desde la observación y la vigilancia entendida ésta última como el esfuerzo sistemático y estructurado de observación, capacitación, análisis, comunicación precisa y recuperación de la información sobre los hechos del entorno económico, tecnológico, social, relevantes para la misma por implicar una amenaza u oportunidad para la misma (Palop y Vicente, 1999).

Entre las razones por las que conviene practicar la vigilancia Hidalgo, León y Pavón (2002) señalan las que siguen:

a) Anticipar. El ejercicio de vigilancia nos permite la constante actualización del conocimiento por lo que nos da la oportunidad de conocer con anticipación los posibles cambios que se están produciendo en el desarrollo científico por lo cual nos permite reforzar la investigación donde ésta es más débil o incorporar nuevas líneas dónde es inexistente.

b) Reducir riesgos: Una consecuencia primera que se convierte en una razón de peso para su práctica es la identificación de campos de investigación 
o de actuación ya resueltos por lo que nos permite planificar la distribución de esfuerzos de manera mucho más rentable y segura.

c) Innovar. Otra de las consecuencias y razones de peso en su práctica es la posibilidad de decidir desde la información reunida el rumbo de cualquier política o actuación. En definitiva, la observación y la vigilancia representan un instrumento de apoyo para cualquier estrategia.

d) Cooperar. Ya he dicho más arriba que uno de los rasgos de la prospectiva es la mutidisciplinaridad; pues bien, la vigilancia permite la identificación de instituciones, líneas y grupos de investigación así como expertos en las diferentes áreas de interés para el ejercicio de la prospectiva.

Desde estas cuatro razones podemos afirmar que la observación y la vigilancia son, en realidad, el punto obligado de partida de todo ejercicio de prospectiva.

\section{TABLA V}

Ciclo del proceso de observación y vigilancia

\begin{tabular}{|c|c|c|c|}
\hline \multirow{5}{*}{$\begin{array}{l}\text { ENTORNO } \\
\text { Archivos y salud } \\
\text { Archivos y justicia } \\
\text { Archivos y cultura } \\
\text { Archivos y economía } \\
\text { Archivos y defensa } \\
\text { Archivos y seguridad } \\
\text { Archivos y trabajo } \\
\text { Archivos y medioambiente }\end{array}$} & 1. & \multicolumn{2}{|c|}{ Identificar las necesidades de información } \\
\hline & 2. & $\begin{array}{l}\text { Buscar } \\
\text { Descubrir } \\
\text { Detectar } \\
\text { Observar } \\
\text { Recolectar } \\
\text { Captar }\end{array}$ & LA INFORMACIÓN \\
\hline & 3. & \multicolumn{2}{|l|}{$\begin{array}{l}\text { Analizar } \\
\text { Tratar } \\
\text { Almacenar }\end{array}$} \\
\hline & 4. & \multicolumn{2}{|c|}{$\begin{array}{l}\text { Dar valor } \\
\text { Convertir la información en inteligencia }\end{array}$} \\
\hline & 5. & \multicolumn{2}{|c|}{ Comunicar la inteligencia } \\
\hline
\end{tabular}

Fuente: Elaboración Propia desde Sánchez, J. M. y Palop (2002).

\subsection{El proceso de la investigación prospectiva}

El de la prospectiva, como cualquier proceso de investigación, abarca diferentes momentos que se enmarcan entre dos momentos clave: la planificación o definición de objetivos y la comunicación o difusión de los resultados. Entre ambos se inscriben: la búsqueda de la información, su análisis, la implementación del ejercicio prospectivo desde la anticipación y el consenso y la elaboración del informe y su comunicación que dará lugar, en su caso, a la toma de decisiones, principalmente en el caso de la prospectiva estratégica. En relación con las fases 
y objetivos en cada una de ellas, el cuadro que sigue recoge las técnicas que propone M. Godet (2000) para la prospectiva estratégica.

TABLA VI

Proceso y técnicas de la prospectiva estratégica según Godet

\begin{tabular}{|c|c|c|}
\hline Fase & Objetivo & Técnica \\
\hline 1 & Iniciar y simular el conjunto del proceso. & $\begin{array}{l}\text { - El método de escenarios. } \\
\text { - Los talleres de prospectiva estratégica. }\end{array}$ \\
\hline 2 & Establecer el diagnóstico completo. & $\begin{array}{l}\text { - Los árboles de competencias. } \\
\text { - Los útiles de análisis estratégico. } \\
\text { - El diagnostico estratégico. }\end{array}$ \\
\hline 3 & $\begin{array}{l}\text { Plantear las buenas preguntas e identificar } \\
\text { la variable clave. }\end{array}$ & - El análisis estructural. \\
\hline 4 & Analizar las estrategias de actores. & - El método Mactor. \\
\hline 5 & $\begin{array}{l}\text { Explorar el campo de los posibles y redu- } \\
\text { cir la incertidumbre. }\end{array}$ & $\begin{array}{l}\text { - El análisis morfológico. } \\
\text { - Método DELPHI. } \\
\text { - El ábaco de Regnier. } \\
\text { - Impactos cruzados probabilizados smic- } \\
\text { prob-expert. }\end{array}$ \\
\hline 6 & $\begin{array}{l}\text { Evaluar las elecciones y las opciones estra- } \\
\text { tégicas. }\end{array}$ & $\begin{array}{l}\text { - Los árboles de pertinencia. } \\
\text { - Multipol. }\end{array}$ \\
\hline
\end{tabular}

Fuente: elaboración propia desde Godet, M. (2000).

Veamos ahora el planteamiento de las principales herramientas en la prospectiva archivística.

\subsection{Herramientas metodológicas}

\section{A. Análisis de tendencias de futuro}

Comprensión del modelo de desarrollo archivístico que lógicamente debe atender a las tendencias de futuro (figura 1).

\section{B. PEST}

Acrónimo para referirse a los aspectos Políticos, Económicos Sociales Tecnológicos que afectan a una institución (Chaptman, 2004). Para el estudio PEST del archivo resulta obligado identificar las tendencias y aspectos en la evolución de la tecnología y la sociedad que han influido, que están influyendo y que influirán sobre el archivo: 
FIGURA 1

Esbozo de un posible modelo de análisis del desarrollo archivístico

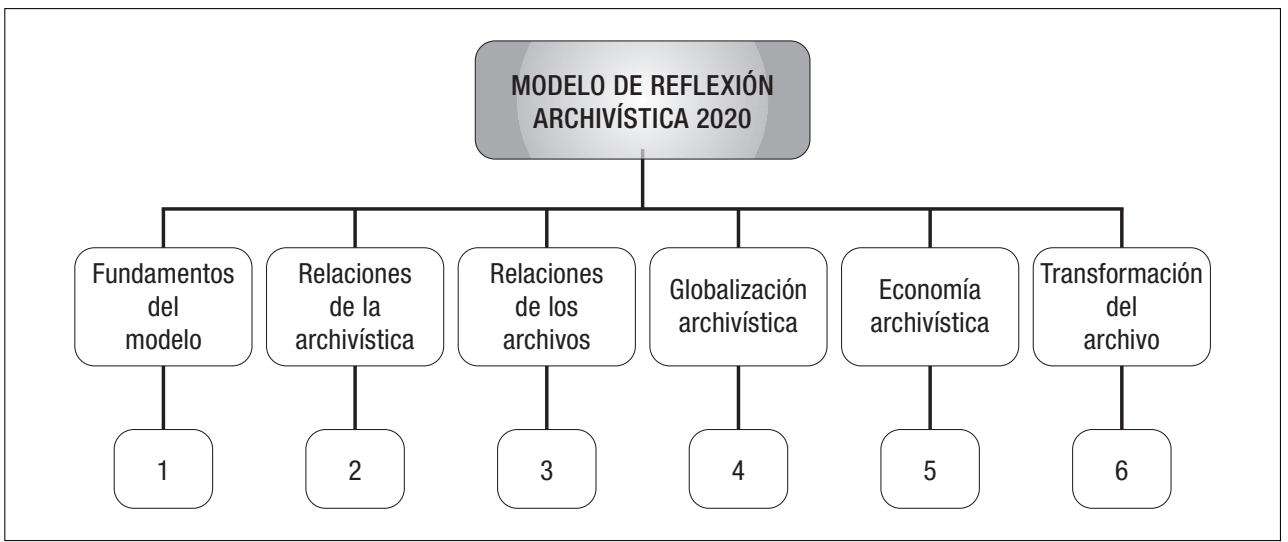

- Tecnología: ¿tele archivo? ¿tele archivística? ¿tele archiveros?

- Organización de la sociedad: ¿la misma estructura administrativa? ¿la misma organización? ¿la misma necesidad del archivo?

- Ética social: ¿las mismas normas sociales? ¿las mismas normas de acceso? ¿de transparencia?

- Globalización: ¿las mismas relaciones interna y externas del archivo?

- Demografía: ¿en la medida que crezca o decrezca la población crecerá o decrecerá el volumen de actividad administrativa? y en esa misma medida ¿crecerá o decrecerá el volumen de los archivos, con independencia del soporte?

\section{Estudio de factores críticos: análisis DAFO, DELPHI}

Para el estudio de los factores críticos / claves que van a marcar el futuro de la disciplina (archivística y gestión de documentos), su objeto de estudio (archivo, información archivística y memoria) los actores involucrados (productores, profesionales y usuarios) la prospectiva archivística dispone de las técnicas denominadas de consenso.

\section{Proceso para el consenso}

Sea cual sea la técnica y la herramienta elegida, el proceso que permite alcanzar un consenso pasa necesariamente por las cinco siguientes fases, a saber:

FASE A: Exploración del tema en discusión. El equipo investigador define el tema, reúne toda la información, define los problemas, cuestiones o ideas sujetas a consenso. En base a esta información identifica el grupo de expertos. 
FASE B. Comunicación con los expertos. Comunicación con este grupo de quien solicita su compromiso a permanecer a lo largo de toda la investigación. Si es el caso facilita toda la información reunida en la fase A.

FASE C. Envío de la información previamente sistematizada y organizada a los diferentes expertos que de manera individual valorará esa información. El objetivo es concretar acuerdos y desacuerdos con respecto al tema y las cuestiones objeto de consensos.

FASE D. Análisis de los desacuerdos: razones y evaluación de las diferencias (esta fase puede implicar nuevos envíos y nuevas evaluaciones hasta llegar al consenso).

FASE E. Elaboración del documento de consenso. Una vez finaliza la fase D.

Especialmente útiles resultan las técnicas DAFO y DELFI. Pasamos a continuación a ilustrar la primera como ejemplo de técnica de consenso. Se presenta con ejemplos claros para la disciplina que proponemos: la prospectiva archivística. Algunos de los rasgos que tienen en común:

- Anonimato. Se evita el contacto entre los participantes.

- Reiteración. El "documento" propuesto estará circulando el tiempo necesario hasta alcanzar el consenso

- Realimentación. A los encuestados se les proporcionan los resultados de las rondas previas.

- Agregación. Los resultados se deben presentar en términos numérico-estadísticos.

\section{Análisis DAFO}

Las siglas DAFO aluden a Debilidades, Amenazas, Fortalezas y Oportunidades de una institución, cultura, política, etc. (véase tabla VII). Su objetivo consiste en:

a) concretar los puntos fuertes y débiles de esa empresa, institución, cultura, política, etc. así como las amenazas y oportunidades externas desde la evaluación externa por expertos, y

b) concretar amenazas y oportunidades para su desarrollo externo.

\section{Elaboración de escenarios de futuro para el archivo}

El método de escenarios es quizá uno de los métodos más y mejor estudiados (Schwartz, 1993; Van der Heijden, 1996; Godet y Roubelat, 1996; Ringland, 1998).

Con Godet entendemos por escenario el conjunto formado por la descripción de una situación futura y un camino de acontecimientos que permiten pasar de una situación original a otra futura. En este sentido, hay que tomar en consideración que el futuro es múltiple pero que además los futuros posibles o futuribles son variados. Cada uno de estos futuribles representan un escenario posible que, 
TABLA VII

Ejemplos de factores claves (DAFO) para la archivística

\begin{tabular}{|c|c|c|}
\hline & Amenazas & Oportunidades \\
\hline \multirow[t]{2}{*}{$\begin{array}{l}\text { Análisis } \\
\text { del entorno } \\
\text { o factores } \\
\text { externos }\end{array}$} & $\begin{array}{l}\text { - Aparición e incremento de otros } \\
\text { profesionales con interés por el ar- } \\
\text { chivo. } \\
\text { - Baja oferta formativa en archivística. } \\
\text { - Baja oferta formativa especializada. } \\
\text { - Plantillas profesionales poco o mal } \\
\text { definidas. } \\
\text { - Escaso reconocimiento del profe- } \\
\text { - Eional. } \\
\text { - Escaso reconocimiento social. } \\
\text { - Eolíticas archivísticas insuficientes. } \\
\text { - Cambio en las necesidades de los } \\
\text { - usuarios. } \\
\text { - Tecnologías cambiantes. }\end{array}$ & $\begin{array}{l}\text { - Nuevos y diversificados espacios de } \\
\text { actuación: la memoria. } \\
\text { - Mayor comprensión social. } \\
\text { - Mayor impacto social. } \\
\text { - Nuevos usuarios de archivos. } \\
\text { - Usuarios más cualificados. } \\
\text { - Mejores instalaciones. } \\
\text { - Crecimiento de plantillas. } \\
\text { - Eliminación de barreras en la rela- } \\
\text { ción con la sociedad. } \\
\text { - Etc. }\end{array}$ \\
\hline & Debilidades & Fortalezas \\
\hline $\begin{array}{l}\text { Diagnóstico } \\
\text { o factores } \\
\text { internos }\end{array}$ & $\begin{array}{l}\text { - Escasez de recursos materiales. } \\
\text { - Escasez de recursos humanos. } \\
\text { - Alta exigencia de especialización. } \\
\text { - Falta de planificación estratégica. } \\
\text { - Incapacidad de financiar los cam- } \\
\text { bios necesarios en la estrategia. } \\
\text { - Atraso en I+D. } \\
\text { - Atraso en I+T. } \\
\text { - Instalaciones obsoletas. } \\
\text { - Etc. }\end{array}$ & $\begin{array}{l}\text { - Capacidad en actividades claves: la } \\
\text { recuperación de la memoria, la his- } \\
\text { toria narrada, la microhistoria. } \\
\text { - Recursos financieros adecuados. } \\
\text { - Habilidades y recursos tecnológicos } \\
\text { - Superiores } \\
\text { - Software libre cada vez más espe- } \\
\text { - ializado. } \\
\text { - Importante programa I+D. } \\
\text { - Buena imagen entre los usuarios. }\end{array}$ \\
\hline
\end{tabular}

en absoluto, mide probabilidades y sí posibilidades. Los escenarios pueden representar una apuesta o referencia o por el contrario una alarma. Para la elaboración de escenarios de futuro siguiendo a Godet contamos con diferentes herramientas en relación con objetivos distintos (véase tabla VIII).

\section{Proceso para la creación de escenarios de futuro}

El proceso para la definición de escenarios de futuro pasa por tres grandes fases:

FASE A: El objetivo de la primera no es otro que definir tendencias de futuro respecto del área o problema objeto de estudio: la archivística como disciplina, la orientación o retos de sus profesionales, sus relaciones con la sociedad, la 
TABLA VIII

Método de escenarios de futuro

\begin{tabular}{l|l}
\hline \multicolumn{1}{c|}{ Denominación } & \multicolumn{1}{c}{ Breve descripción } \\
\hline Talleres de prospectiva & El problema expuesto, el sistema estudiado. \\
\hline Análisis estructural & Búsqueda de factores y variables clave. \\
\hline $\begin{array}{l}\text { Análisis de estrategias de actores } \\
\text { Método MACTOR }\end{array}$ & Juegos y objetivos estratégicos. \\
\hline $\begin{array}{l}\text { Análisis morfológico } \\
\text { Método MORPHOL }\end{array}$ & Balizar el campo de los posibles. \\
\hline $\begin{array}{l}\text { Método de expertos } \\
\text { Encuesta SMIC } \\
\text { Prob-Expert }\end{array}$ & $\begin{array}{l}\text { Cuestiones clave para el futuro. } \\
\text { Juego de hipótesis realizadas. }\end{array}$ \\
\hline
\end{tabular}

Fuente: Elaboración propia desde Godet (2002)

orientación de las políticas públicas para archivos, etc. Para ello tendremos antes de nada que identificar de la manera más exhaustiva posible todos los aspectos que estén ya afectando o vayan a afectar ese objeto de análisis. Esta información será el resultado del ejercicio previo de vigilancia. En ocasiones y cuando se trate de asuntos completamente nuevos para reunir la información necesaria puede recurrirse a la colaboración de expertos. El resultado de esta fase debe ser un listado de tendencias que luego serán discutidas.

FASE B. El objetivo de esta segunda fase será definir las relaciones existentes entre las distintas tendencias o variables. Para ello se deben sistematizar estas variables de acuerdo con criterios previamente definidos. En todo caso siempre se incluirá el impacto en el futuro y el grado de incertidumbre de estas variables.

FASE C. En esta fase tiene ya lugar el diseño del o los escenarios posibles. La cuestión que reviste en esta fase mayor dificultad es la de decidir el numero de escenarios. En la literatura no existe un numero idóneo, en realidad la mejor ruta parece la de orientar la selección en función de los temas implicados, con la siguiente combinación por orientación de calidad del mismo.

FIGURA 2

Esquema de escenarios básicos en el desarrollo de la archivística

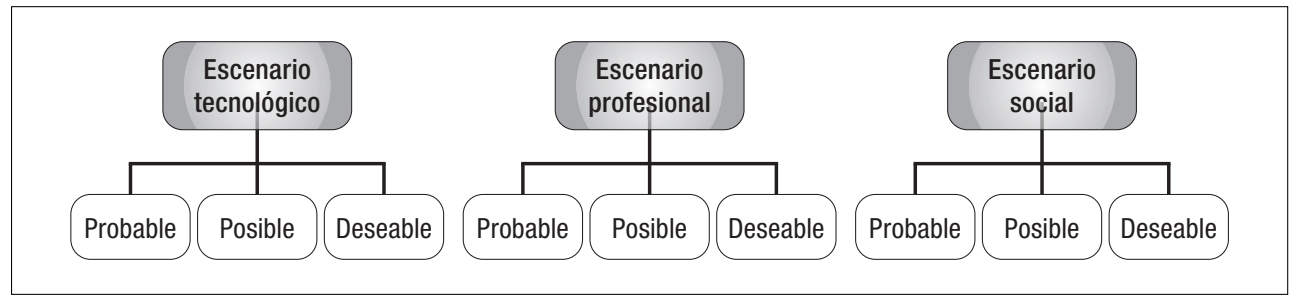




\section{Resultados que la prospectiva archivistica puede generar}

El resultado del proceso de la prospectiva archivística será, por tanto, el conjunto de escenarios, estrategias y recomendaciones adaptadas a los retos y desafíos futuros de la archivística, de los archivos, de los archiveros así como un conjunto de principios y estrategias de respuesta para la integración y la convivencia de los archivos en la sociedad, en todos los espacios definidos: la ciudad, el ayuntamiento, la justicia, la seguridad, la sanidad, la universidad, etc.

Para cada uno de ellos, además, se concretan dos grupos de resultados específicos:

a) Los documentos de consenso: la elaboración de normas o recomendaciones de índole diagnóstica y/o terapéutica que expresen de forma consensuada y oficial la opinión de los expertos en el análisis de diferentes problemas y cuestiones. También servir de instrumento conceptual y metodológico para el manejo de estas situaciones.

b) Los indicadores: estadísticas o cualquier forma de indicación que nos permitan estudiar dónde estamos y hacia dónde nos dirigimos con respecto a determinados objetivos y metas. Que nos permitan también evaluar programas, proyectos, sistemas, etc.

\section{Conclusiones}

1. La archivística fuertemente influenciada por los cambios sociales y tecnológicos está viendo modificada su naturaleza, en consecuencia le urge: la revisión de su construcción teórica y la revisión de los mecanismos de su metodología.

2. Ninguna de estas revisiones sería posible sin analizar con la suficiente profundidad las cuestiones y las incertidumbres a las que se enfrenta la disciplina en el futuro más y menos inmediato.

3. Ante esta situación la prospectiva es la investigación que por el momento nos permite conocer los factores y puntos clave de ese futuro, así como la herramienta metodológica que facilita y hace posible la reflexión colectiva sobre el archivo, la archivística y sus profesionales.

4. En su relación con la archivística la prospectiva es una metodología cuyos rasgos podemos concretar del modo que sigue: se fundamenta en la observación sistemática, la vigilancia y la inteligencia competitiva; se construye desde el debate y el consenso; facilita y sistematiza la reflexión colectiva.

5. Para su aplicación y análisis requiere de la colaboración de diferentes áreas de conocimiento, lo que la dota de un carácter multidisciplinar, facilita la anticipación y está referida a largo plazo. 
6. La investigación prospectiva nos permite $a$ ) la identificación de variables clave para el futuro de los archivos; $b$ ) el análisis de tendencias de futuro en relación con las variables o factores clave; c) análisis prospectivos parciales: dinámicas demográficas, futuro de los profesionales, de la enseñanza, de la investigación, de la formación, potencial de los archivos, innovación en los archivos, política archivística, redes y colaboración entre archiveros.

\section{Bibliografia}

Ackoff, R. (1979): Rediseñando el futuro. México: Limusa.

Bailly, J. P. (1998): Prospective, débat, decision publique. Avis du Conseil économique et social. Futuribles, 10, 27-51.

Bell, D. (1976): El advenimiento de la sociedad post-industrial. Madrid; Alianza Universidad, 149

Bell, W. (1997): Foundations of futures studies. London; Transaction Publishers.

Berger, G. (1957): Sciences humaines et prevision. La revue de deux monde, 3, 417-426.

Borglund, E. (2005): Operational use of electronic records in police work. Information Research an international journal, vol. 10. No. 4. Accesible en: http://informationr. net/ir/10-4/paper236.html [consultado el 8 de abril de 2009].

Bourg, D., y Scheler, J. L. (2001): Oarer aux risqué de demain. Le principe de precaution. Paris; Editions du Seuil.

Cacace, N. (2002): 2010, scenario delle professione. Roma: Edit, Riunti.

Cook, T. (2001): Archival science and postmodernism: new formulations for old concepts. Archival Science, 1 (1), 3-24.

Clarke, R.; Durand M.; Pilat, D., y Torres, R. (2001): La nouvelle economie: mythe o realité. Le rapport du OCDE sur la croissance. París: OCDE.

Chaptman, A. (2004): Análisis DOFA y análisis PEST. Accesible en: http://www.degerencia. com/articulos.php?artid=544 [consultado el 8 de abril de 2009].

Duranti, L. (2000): The impact of technological change on archival theory. International Council on Archives, XIV Congreso, Sevilla, 39-55.

Drucker, P. F. (2003): El management del futuro. Buenos Aires; Editorial Sudamericana.

Flechtheim, O. K. (1966): History and futurology. Meisenheim am Glan: Hain.

García, E. (2004): Medio Ambiente y sociedad. La civilización industrial y los límites del planeta. Madrid: Alianza Editorial.

Gallisot, J. (2004): Quelle planéte Terre pour les generations futures? París: Publibook.

Gavigan, J. P. (2001): Panorama de la prospectiva en Europa. Principios y visión general por países. Economía industrial, 342, VI, 107-115.

Gilfillan, S. C. (1907): The Sociology of Invention. Cambridge: MIT Pres.

Gilliland-Swetland, A. J. (2000): Enduring paradigm, new opportunities: the value of the archival perspective in the digital environment. Washington, D.C.: Council on Library and Information Resources. 
Godet, M. (2004): Manuel de prospective estratégique. T1: une discipline intellectuel. Paris: DUNOD.

Godet, M. (2000): La caja de herramientas de la prospectiva estratégica. Zarauz, Guipuzcoa; Prospeptiker, cuaderno $\mathrm{n}^{\circ} 5$.

Godet, M., y Roubelat, F. (1996): Creating the future: the use and misuse of scenarios, Long range planning, vol. 29, $\mathrm{n}^{\circ} 2,164-171$

Healy, T.; Coté, S., y otros (2001): Du bien être des nations. Le roir du capital humain et social. Paris: OCDE.

Heilbroner, R. (1996): Visiones del futuro: el pasado lejano, el ayer, el hoy y el mañana. Barcelona; Ediciones Paidós Ibérica.

Hidalgo, A.; León, G., y Pavón, A. (2002): La gestión de la innovación y las tecnologías en las organizaciones. Madrid: Pirámide.

Jacquard, A. y Khan, A. (2001): L'avenir n'est pas écrit. Paris: Bayard.

Jancovici, J.M. (2002): L'avernir climatique ¿quell temps feron nous? París: Editions du Seuil.

Janstch, E. (1970): Pronósticos del futuro. Madrid: Alianza Editorial.

Jouvenel, B. (1967): The Art of Conjecture. New York: Basic Books.

Leff, E. (2004): Ecología y Capital. Racionalidad ambiental, democracia participativa y desarrollo sustentable. Ed. Siglo XXI en coedición con el Instituto de Investigaciones Sociales de la UNAM: México.

Martín-Pozuelo, M. P. (2002): Los entornos y documentos electrónicos ¿Efecto Edison para la formación archivística?. V Jornadas de Archivos Electrónicos. Priego de Córdoba, 51-64.

Masini, E. B. (2000): Penser le futur : les bases des études prospectives. París: Dunod éditeur.

Medina Vásquez, J., y Ortegón, E. (2006): Manual de prospectiva y decisión estratégica: bases teóricas e instrumentos para América Latina y el Caribe. Santiago de Chile: IELS, Manuales $\mathrm{n}^{\circ}$ 51, 138.

Michalski, W., y Miller, R. (1999): L'economie mundiale du demain. Vers un essors durable. Paris: OCDE.

Palop, F., y Vicente, J. M. (1999): Vigilancia tecnológica e inteligencia competitiva. Su potencial para la empresa española. Fundación COTEC, Estudio 15.

Pérez Andrés, C. (2000): ¿Deben estar las técnicas de consenso incluidas entre las técnicas de investigación cualitativa? Revista Española de Salud Pública, 74, n 4, 319-321.

Polak, F. (1971): The image of the future. Amsterdam: Elsevier Scientific Publishing Company.

Ringland, G. (1998): Scenario Planning: Managing for the Future. Chichester: John Wiley \& Sons,

Serra, J. (1997): "Imaginar un mañana”. La Vanguardia [en linea]. Ciencia y salud. Disponible en: http://www.cienc.ia.vanguardia.es/ciencia/portada/p371.html [consultado el 6 de mayo de 2009].

Sherman, K. (1994): Inteligencia estratégica para la política mundial norteamericana. Buenos Aires: Editorial Pleamar.

Schwartz P., (1993): La planification stratégique par scénarios, Futuribles, n $176,31-50$ 
Van Der Heijden, K. (1996): Scenarios: The Art of Strategic Conversation. Chichester \& New York: John Wiley \& Sons.

Vendramin, P., y Valenduc, G. (2002): L'avenir du travail dans la societé de l'information. Enjeux individuels et collectives. Paris: L'hartman.

Waardenburg, J. (1999): Classical Approaches to the Study of Religion: Aims, Methods, and Theories of Research. New York (etc.) Walter de Gruyter.

Wallerstein, I. (2002): Un mundo incierto. Buenos Aires: libros del Zorzal. 